\title{
Praxissoftware
}

\section{Medistar bleibt Spitzenreiter}

\section{In der Rangliste der Praxis-EDV-Anbieter - zumindest in den Top Ten - hat sich wenig geändert. Aber erstmals ist die Zahl der Installationen rückläufig.}

Eine Arztpraxis ohne EDV-System, das scheint eher eine Seltenheit zu sein, betrachtet man die aktuelle Installationsstatistik der Kassenärztlichen Bundesvereinigung (KBV). Denn die KBV meldet insgesamt 115440 Installationen von Praxis-EDV-Systemen (Stand 30.06.2010).

Allerdings ist zum ersten Mal seit langem die Gesamtzahl der Installationen gesunken, und zwar um 2133 im Vergleich zum Vorjahr (damals zählte die KBV 117573 Installationen).

Woran das liegt, darüber kann man bislang nur rätseln. Ein Grund könnte ein steigender Kooperationsgrad sein. Die Anzahl der Vertragsärzte hingegen ist eher nicht gesunken. Zumindest nach den aktuellsten Zahlen der KBV, die allerdings nur einen Vergleich der Zahl der Vertragsärzte in 2009 zu 2008 zulassen. Hier gab es einen leichten Zuwachs: Von 136063 auf 137128 Vertragsärzte und -psychotherapeuten.

\section{CompuGroup besonders erfolgreich}

Unter den Top Ten der Praxis-EDV-Anbieter gibt es auch wie im Vorjahr kaum Veränderungen. An erster Stelle steht die CompuGroup mit insgesamt 21843 Installationen, gefolgt von medatiXX (21 117) und TurboMed (13 365), die ebenfalls zur CompuGroup gehören. Auf Rang sieben folgt die Frey ADV GmbH, das unabhängige Softwarehaus Duria eG erreicht dieses Mal Rang 13.

Das beliebteste EDV-System unter niedergelassenen Ärzten ist weiterhin Medistar, ebenfalls ein Unternehmen der CompuGroup. Dabei konnte Medistar im Vergleich zum Vorjahr seinen Marktanteil leicht auf 12,58\% erhöhen. Insgesamt erreichte die Software 14524 Installationen, das sind 38 mehr als im Vorjahr. Auf dem zweiten Platz ist TurboMed mit 13365 Installationen und einem Marktanteil von 11,58\%, gefolgt von MCS-ISYNET mit 9022 Installationen und Albis on Windows mit 7319 Installationen. Ebenfalls in die Top Ten schafft es Quincy Win von Frey ADV, und zwar auf Platz zehn mit 4169 Installationen. Betrachtet man nur die Facharztgruppe der Allgemeinärzte, dann liegt TurboMed mit 4234 Installationen auf Platz eins, gefolgt von Medistar mit 3005 Installationen und DOCcomfort von medatiXX mit 2467.
Kurz notiert

\section{DGN bietet neuen KV-SafeNet-Router an}

Das Deutsche Gesundheitsnetz (DGN), das zum IT-Anbieter DGN Service $\mathrm{GmbH}$ gehört, hat einen neuen Zugangsrouter für das KV-SafeNet entwickelt. Das Eigenprodukt „GUSbox ${ }^{\circledR} \mathrm{S}^{\text {“ }}$ löse die bislang vom DGN konfigurierte FRITZ!Box als KV-SafeNet-Router ab, meldet das Unternehmen, und werde als Mietgerät für monatlich 4,90 Euro angeboten. Im Mietpreis enthalten seien die Firmware, Sicherheits-Updates sowie ein 24-Stunden-Austausch-Service im Fall einer defekten Box. Zudem soll die GUSbox S im zweiten Quartal mit zusätzlichen Diensten, etwa einem eingebauten D2D-Dienst, ausgestattet werden.

\section{promedico relauncht eigene Homepage}

Die promedico Computer für Medizin $\mathrm{GmbH}$ hat einen neuen Internetauftritt. Die Inhalte der neuen Webseite reichen von aktuellen Informationen über Produktbeschreibungen bis hin zu Serviceangebote für die Anwender einer promedico-Software, so das Unternehmen.

Beispielsweise sollen Besucher der Seite zu aktuellen Themen wie den Ambulanten Kodierrichtlinien umfassende Informationen erhalten. Außerdem sollen promedico-Kunden Hilfe, Unterstützung und Informationen zu Produktneuheiten finden. In einem FAQ-Bereich werden, nach Angaben von promedico, oft gestellte Fragen beantwortet. Interviews mit und Berichte von promedico-Anwendern sollen den Praxisteams Aufschluss darüber geben, welche Erfahrungen Kollegen mit promedicoLösungen gesammelt haben. Zudem sollen regelmäßige und aktuelle Informationen auch über einen Newsletter bezogen werden können. eb www.promedico.com

\section{Studie sieht Roche bei Websites auf Platz eins}

Das Pharma-Unternehmen Roche hält im Internet das beste Angebot für medizinische Fachkreise vor. Das hat die Studie Benchmarking Websites Pharma 2010 ergeben. Die Studie ist jetzt zum zehnten Mal vom Unternehmen Consert Online Service Pharma vorgelegt worden. Auf den Plätzen zwei bis fünf folgen mit ihren Web-Angeboten für Fachkreise die Unternehmen Bayer Vital, Hexal, GlaxoSmithKline und MSD Sharp \& Dohme. Mehr als 4000 medizinische Websites von PharmaUnternehmen in insgesamt 18 Indikationsbereichen sind anhand eines einheitlichen Rasters in die Bewertung eingeflossen.

\section{Experten erwarten hohe Nachfrage bei Tablet-PC}

Die Zahl der weltweit verkauften Tablet-Computer wird sich nach Einschätzung der Marktforschungsfirma IDC bis 2012 auf 70,8 Millionen mehr als vervierfachen. Die IDC-Experten liegen damit zwischen den jüngsten Prognosen der Marktforscher von Forrester und Gartner.

Angetrieben von der hohen Nachfrage nach dem iPad von Apple ist der Verkauf von Tablet-PC laut einer am 18. Januar veröffentlichten Studie allein vom zweiten zum dritten Quartal 2010 um 45,1\% auf 4,8 Millionen Geräte gestiegen. Davon entfielen $87,4 \%$ auf das iPad. Zusätzliche Impulse erwartet IDC von der Einführung des Samsung-Modells Galaxy Tab im vierten Quartal des vergangenen Jahres sowie vom Blackberry PlayBook und vom Motorola Xoom in diesem Jahr. Im Schlussquartal 2010 verkaufte allein Apple 7,3 Millionen seiner iPads. Die IDCMarktforscher schätzen die Zahl der 2010 verkauften Tablet-PC auf nahezu 17 Millionen. In diesem Jahr sagen sie einen Absatz von 44,6 Millionen voraus, wobei ein Anteil von rund $40 \%$ allein auf Lieferungen in den USA entfällt. Gartner wagte im Oktober die Prognose von gut 103,4 Millionen verkauften Tablets im Jahr 2012. 